\title{
Muth, Cornelia
}

\section{Interkulturelles Lernen und Forschen in transkulturellen Dialoggruppen}

Spetsmann-Kunkel, Martin [Hrsg.]; Frieters-Reermann, Norbert [Hrsg.]: Soziale Arbeit in der Migrationsgesellschaft. Opladen ; Berlin ; Toronto : Verlag Barbara Budrich 2013, S. 55-63. - (Schriften der Katholischen Hochschule Nordrhein-Westfalen; 17)

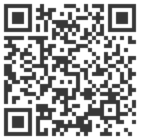

Quellenangabe/ Reference:

Muth, Cornelia: Interkulturelles Lernen und Forschen in transkulturellen Dialoggruppen - In:

Spetsmann-Kunkel, Martin [Hrsg.]; Frieters-Reermann, Norbert [Hrsg.]: Soziale Arbeit in der

Migrationsgesellschaft. Opladen ; Berlin ; Toronto : Verlag Barbara Budrich 2013, S. 55-63 - URN:

urn:nbn:de:0111-pedocs-127811 - DOI: 10.25656/01:12781

https://nbn-resolving.org/urn:nbn:de:0111-pedocs-127811

https://doi.org/10.25656/01:12781

in Kooperation mit / in cooperation with:

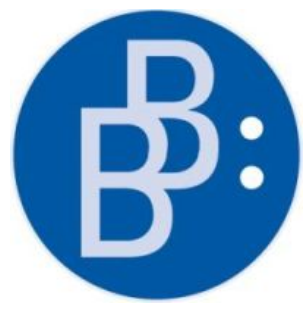

https://www.budrich.de

\section{Nutzungsbedingungen}

Dieses Dokument steht unter folgender Creative Commons-Lizenz:

http://creativecommons.org/licenses/by-nc-nd/3.0/de/deed - Sie dürfen das Werk bzw. den Inhalt unter folgenden Bedingungen vervielfältigen, verbreiten und öffentlich zugänglich machen: Sie müssen den Namen des Autors/Rechteinhabers in der von ihm festgelegten Weise nennen. Dieses Werk bzw. dieser Inhalt darf nicht für kommerzielle Zwecke verwendet werden und es darf nicht bearbeitet, abgewandelt oder in anderer Weise verändert werden.

Mit der Verwendung dieses Dokuments erkennen Sie die Nutzungsbedingungen an.

\section{Terms of use}

This document is published under following Creative Commons-License: http://creativecommons.org/licenses/by-nc-nd/3.0/de/deed.en - You may copy, distribute and transmit, adapt or exhibit the work in the public as long as you attribute the work in the manner specified by the author or licensor. You are not allowed to make commercial use of the work or its contents. You are not allowed to alter, transform, or change this work in any other way.

By using this particular document, you accept the above-stated conditions of use.

\section{Kontakt / Contact:}

peDOcs

DIPF | Leibniz-Institut für Bildungsforschung und Bildungsinformation

Informationszentrum (IZ) Bildung

E-Mail: pedocs@dipf.de

Internet: www.pedocs.de

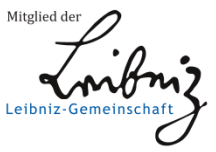


Martin Spetsmann-Kunkel

Norbert Frieters-Reermann (Hrsg.)

\section{Soziale Arbeit in der Migrationsgesellschaft}

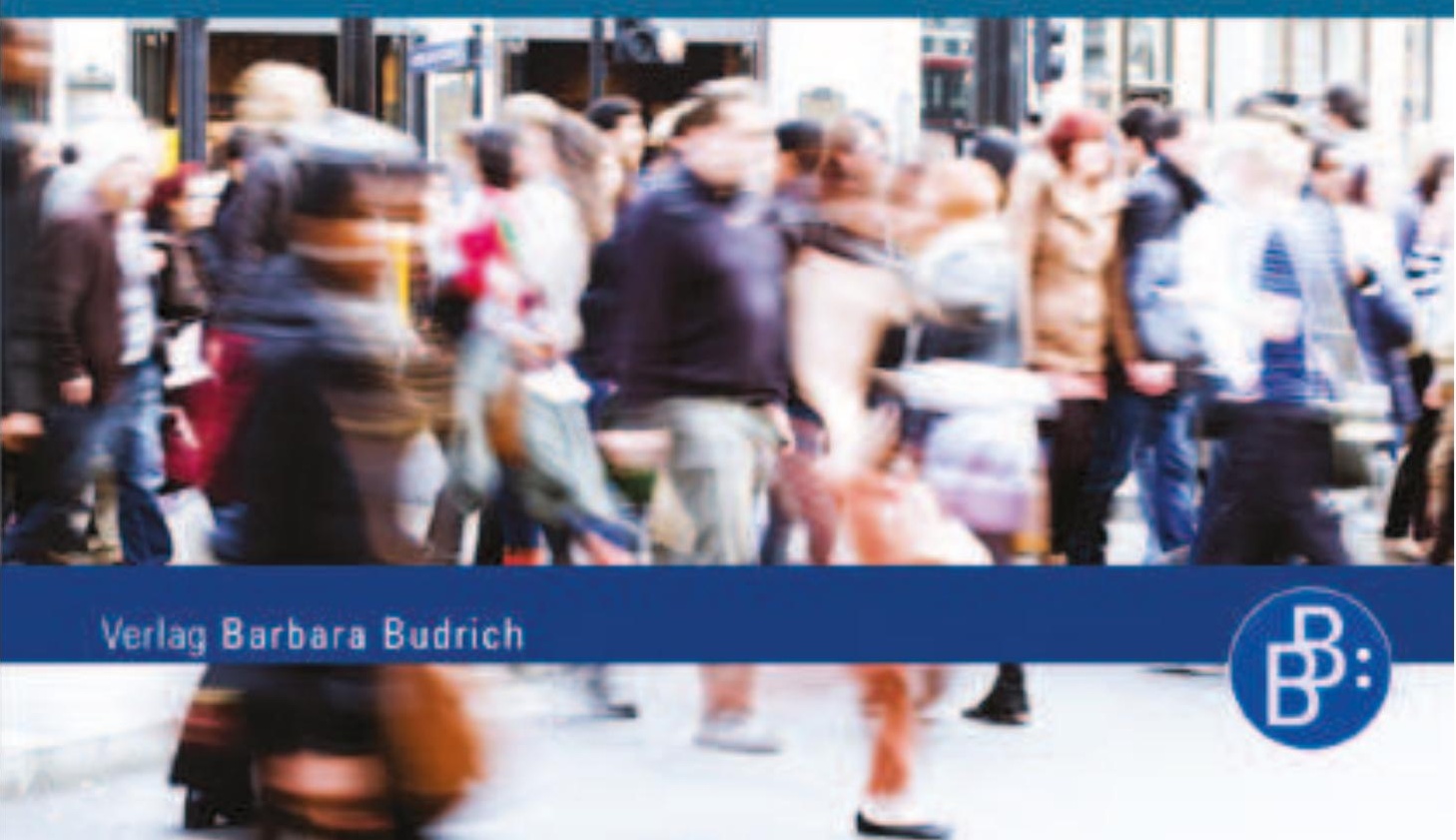


Schriften der Katholischen Hochschule

Nordrhein-Westfalen

Band 17

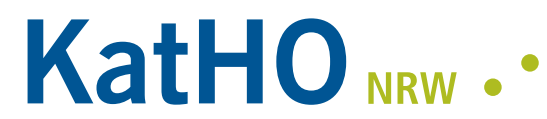

Aachen | Köln | Münster | Paderborn

Katholische Hochschule Nordrhein-Westfalen Catholic University of Applied Sciences 
Martin Spetsmann-Kunkel

Norbert Frieters-Reermann (Hrsg.)

\section{Soziale Arbeit in der} Migrationsgesellschaft

Verlag Barbara Budrich

Opladen, Berlin \& Toronto 2013 
Prof. Dr. Martin Spetsmann-Kunkel

Prof. Dr. Norbert Frieters-Reermann

Katholische Hochschule NRW / Aachen

Robert-Schuman-Straße 25

52066 Aachen

Tel.: (+49) (0)241-6000318

Bibliografische Information der Deutschen Nationalbibliothek

Die Deutsche Nationalbibliothek verzeichnet diese Publikation in der Deutschen

Nationalbibliografie; detaillierte bibliografische Daten sind im Internet über

http://dnb.d-nb.de abrufbar.

(C) 2013 Dieses Werk ist im Verlag Barbara Budrich erschienen und steht unter folgender Creative Commons Lizenz: http://creativecommons.org/licenses/by-nc$\mathrm{nd} / 3.0 / \mathrm{de} /$

Verbreitung, Speicherung und Vervielfältigung erlaubt, kommerzielle Nutzung und Veränderung nur mit Genehmigung des Verlags Barbara Budrich

Dieses Buch steht im OpenAccess Bereich der Verlagsseite zum kostenlosen Download bereit (https://doi.org/10.3224/93809468)

Eine kostenpflichtige Druckversion kann über den Verlag bezogen werden. Die Seitenzahlen in der Druck- und Onlineversion sind identisch.

\section{ISBN 978-3-938094-68-6 (Paperback) \\ eISBN 978-3-8474-0337-1 (eBook) \\ DOI $\quad 10.3224 / 93809468$}

Umschlaggestaltung: disegno visuelle kommunikation, Wuppertal - www.disenjo.de Typografisches Lektorat: Petra Reiners, Bonn - www.buchfinken.com Druck: paper \& tinta, Warschau

Printed in Germany 


\section{Inhaltsverzeichnis}

1. Soziale Arbeit in der Migrationsgesellschaft einleitende Gedanken

Martin Spetsmann-Kunkel

2. Anfragen an die Praxis der Sozialen Arbeit in einer von Mobilität und Vielfalt geprägten Postmoderne - Soziale Arbeit in der Migrationsgesellschaft

Wolf-D. Bukow

3. Interkulturelle Soziale Arbeit: Integration, Anerkennung und Partizipation als Leitideen einer differenzsensiblen Sozialen Arbeit in der Migrationsgesellschaft

Josef Freise

4. Interkulturelles Lernen und Forschen in transkulturellen Dialoggruppen

Cornelia Muth

5. Demokratiepädagogik und Diversity Education pädagogische Konzepte und ihre Bedeutung für die Soziale Arbeit

Schahrzad Farrokhzad 
6. Kritische Soziale Arbeit in Diskriminierungs- und Herrschaftsverhältnissen - eine Skizze

Claus Melter

7. Soziale Arbeit in mobiler Gesellschaft Institutionelle Bedingungen, professionelle Expertise und das eigene Verschränkt-Sein in Alltagsrassismus und kulturalisierenden Deutungsprozessen

Claudia Roller

8. Interkulturelle Öffnung als Team - von Stolpersteinen und Meilensteinen

Norbert Teutenberg

9. Die Migrationssozialarbeit der Caritas

Thomas Kley

10. Soziale Arbeit und Migration - Auslassungen, Anregungen und Ausblicke

Norbert Frieters-Reermann 


\title{
4. Interkulturelles Lernen und Forschen in transkulturellen Dialoggruppen
}

\author{
Cornelia Muth
}

\section{Einleitung}

Eines der Bildungsziele transkultureller Dialogik (Muth 2011) liegt in der Umsetzung, Respekt vor der Andersheit des/der Anderen zu erfahren und zu erleben (vgl. Muth 2008). Das Besondere an transkultureller Dialogik drückt sich zudem in der Wertschätzung des Lebens selbst aus. Letzteres verläuft, wie wir alle jeden Tag neu erfahren können, nicht wissenschaftlich systematisiert, sondern widersprüchlich und kann, aus dialogischer Perspektive, von Situation zu Situation neu betrachtet und neu beschieden werden, auch wenn es sich um eine Wiederholung handelt:

„Ich sage, wo eine Situation einen antrete, da sei es nicht an der Zeit, in einem Wörterbuch nachzuschlagen. Das Bild sollte doch wohl jedem klar sein: im Angesicht der jetzt erscheinenden Situation - nehmen wir zur Verdeutlichung an: einer unvorhergesehenen und unvorhersehbaren Situation - habe ich nicht zu überlegen, welchem Allgemeinprinzip diese Situation zu subsumieren und welches Prinzip somit auf sie anzuwenden sei, sondern es liebt mir ob, mich dieser ,neuen' Situation zu stellen, selbstverständlich: mich mit allem, was ich bin und was ich weiß, zu stellen, und sie im Maße meines Könnens zu bewältigen, also das ihr Angemessene zu tun, ihr zu entgegnen“(Buber 1963, S. 595).

sagt Martin Buber, dessen philosophische Anthropologie Grundlage meiner transkulturellen Dialogtheorie ist. Der Dialogphilosoph Buber (1878-1965) will mit seinen Schriften auf die dialogische Wirklichkeit hinweisen. Für die Soziale Arbeit geht es mir darum, zwischenmenschliche Wahrnehmungen theoretisch wie praktisch zu differenzieren, um damit den dialogischen Raum sozialer Wirklichkeit zu zeigen (vgl. Mührel 2009).

Um interkulturelle Erfahrung und transkulturelle Begegnung $\mathrm{zu}$ unterscheiden, unterscheide ich zwischen interkulturell und transkulturell in Anlehnung an Bubers Beschreibung zur zweifachen Haltung des Menschen zur Welt und zu seinen Mitmenschen. Er versteht darunter einerseits eine IchDu-Haltung und andererseits ein Ich-Es-Verhältnis. Beide zusammen ergeben das Doppelverhältnis des Menschen zum Sein (vgl. Muth 2012a). Jedoch ist mit Es des Ich-Es nicht das Es in der Psychoanalyse Freuds gemeint, sondern Es bezeichnet ein Objekt oder auch eine Theorie und beschreibt das Verhältnis, das das Ich eines Menschen innehält, wenn er/sie einen 
Gegenstand oder eine Methode benutzt. Hier sprechen wir dann von einer Subjekt-Objekt-Beziehung.

In der Ich-Du-Haltung hingegen lässt sich der Mensch auf seinen Mitmenschen ein und von dessen Du berühren. Dann ist das Gegenüber kein Gegenstand mehr, wie beim Ich-Es. Vielmehr geschieht eine SubjektSubjekt-Begegnung. Dem setze ich Ich-Es für eine interkulturelle Betrachtung und Ich-Du für einen transkulturellen Dialog gleich. An einer transkulturellen Begegnung nehmen die Menschen wirklich teil und tun nicht so als ob. Sie wollen nicht gerecht erscheinen, sondern versuchen in aller Widersprüchlichkeit der Anderheit des/der Anderen und dem Leben gerecht zu werden (vgl. Muth 2009b). Buber beschreibt diesen dialogischen Prozess so:

„Du stehst vor eine politischen Entscheidung ... Du bist vom Gebot der Gerechtigkeit getrieben ... Du schonst dich nicht ... Und nun, mitten im Ringen ... geschieht etwas. ..., dass du überraschend, zuweilen geradezu überwältigend merkst, was von deiner Wahrheit und Gerechtigkeit sich in dieser Situation verwirklichen lässt. Du merkst, du hast gemerkt, wieviel dem Leben gegeben werden muss, damit die Gerechtigkeit Leben annehme. Und in eben diesem Moment ... schießen die Kräfte deiner Seele, die eben noch widereinander stritten, zusammen, wie zu einem Kristall schießen sie zusammen“" (Buber 1963, S. 618).

Polar zum dialogischen Geschehen wird interkulturelles Lernen und Forschen als monologische Entwicklung verstanden: Ich mache Erfahrungen mit Menschen und deren Diversität, mit der multikulturellen Welt und mit Theorien über die Welt und über die Menschen. Wenn ich diese wissenschaftlich systematisiere und kritisch reflektiere, bleibt mein Ich in einem Ich-Es-Verhältnis zum Menschen, da ich letzteren einem heteronomen System von Nationalität, Beruf, Heimatland, Geburtstort, Religions- bzw. Ideologiezugehörigkeit, Sexualität als medizinische Kategorie, Einkommen, Wohnort, Auslandserfahrungen, Migration und biographischen Lebenslauf etc. zuordne. Ich mache damit mein Gegenüber zu einem Objekt, das ich erklären und verstehen kann. Transkulturelle Dialogik erkennt dieses Vorgehen als eine Seite (wissenschaftlicher und pädagogischer) Lebenspraxis an, geht jedoch davon aus, dass daraus allein kein Lebenssinn und keine lebensnahe Entscheidung über Soziale Arbeit in der Migrationsgesellschaft allein getroffen werden kann:

„Die Verantwortung vor einer Idee ist fiktiv, wie die Idee mich nicht zur Verantwortung ziehen kann, weil sie nicht entscheiden kann, ob meine Verantwortung zu Recht oder zu Unrecht besteht. Ich verlange einem Begriff wie Verantwortung seinen ursprünglichen konkreten Sinn ab, ich gebe nicht zu, dass ihm ermöglicht werde, sich unterm Schutz einer Philosophie in die Innerlichkeit zu verflüchtigen“ (Buber 1963, S. 596). 
Infolgedessen erweitert transkulturelle Dialogik die Perspektive auf das Ich$\mathrm{Du}$ im Sinne einer Subjekt-Subjekt-Begegnung: Eine sich gegenseitig anerkennende Beziehung kann abseits von machtvollen Begriffen möglich werden. So drückt eine transkulturelle Begegnung im gegenwärtigen Moment zwischenmenschliches Vertrauen unabhängig von Rollen und Funktionen aus. Das, was in der Gegenwart geschieht und überraschend neu wahrgenommen wird, lässt Veränderung zu. Dabei bleiben Ich und mein Gegenüber systematisch unbestimmt und weder das Ich noch das Du können empathisch einverleibt werden, denn ich kann nicht fühlen, was der/die Andere fühlt. Doch können wir, das Ich und Du, uns gegenseitig gewahr werden.

\section{Zum Kulturbegriff transkultureller Dialogik}

Als Objekt transkultureller Bewusstseinsprozesse bedeutet dann interkulturelles Lernen, Kultur als Begriff für eine „Vielzahl von Lebensformen“ zu verstehen (vgl. Muth 2011, S. 18ff.). Dabei ist jede Lebensform wiederum an eine soziale Struktur mit einer spezifischen und einzigartig individuellen Lebenspraxis verbunden. Die Vielzahl dieser Lebensformen ist mehr oder weniger inkommensurabel, d.h. dialogisch gesehen, nicht in einem Ich-EsSystem, in einer sozialen Hierarchie erfassbar. Die „Vielzahl der Lebensformen" versucht als Kulturbegriff zu beschreiben, dass jede Lebensform sich in einem rhizomartigen Werdensprozess befindet, bei dem von grundsätzlichen Hegemonien in Hinblick auf die Andersheit des/des Anderen ausgegangen wird (vgl. Deleuze/Guattari 1977). Deswegen muss ich hier einwandfreier von einer unendlichen Gerechtigkeit sprechen, die wir als Dialogpädagog/innen verantworten. Christina Thürmer-Rohr (2003) spricht desgleichem vom „Dialog als unendlichem Aufklärungsexperiment“. Wir verwirklichen durch den Dialog das demokratische Prinzip des Dissenses in der Pluralität. Es betont im mehrfach differenzierten Nebeneinander die Vielzahl von Lebensformen. Für Michael Göhlich (2009, S. 147) ist diese Perspektive Teil einer spezifisch pädagogischen Professionalität, die Differenz ermöglicht:

„Differenz meint hier nicht nur, möglicherweise sogar nicht einmal in erster Linie Unterschied, sondern vor allem (die Erfahrung von) Grenze. Die Erfahrung des Andersseins, des Nicht-Verstanden-Werdens, aber auch des den-Anderen-nicht-Verstehens birgt Schmerz. Differenz ist in der pädagogischen Praxis als Schmerz(-grenze) wahrzunehmen und zu achten. Die Anerkennung des Schmerzes der Differenz ist ein Baustein zur Anerkennung des Anderen." 
Solche und andere Differenz-Erfahrungen sind in transkulturellen Dialoggruppen möglich (vgl. Muth 2010, 2009a, 2005a, 2005b).

\section{Das Setting einer transkulturellen Dialoggruppe}

$\mathrm{Zu}$ Beginn jedes Dialoggruppenprozesses, sei es für einen einzigen Workshop oder für ein akademisches Semester, lege ich die Orientierungskriterien dar und erläutere sie:

- $\quad$ Eine Gruppe trifft sich regelmäßig und die Teilnahme ist freiwillig.

- Das Ziel ist das Erreichen eines wirklichen Gruppengesprächs, das sich darin zeigt, dass Wahrnehmungen zusammengebracht werden.

- Das Einlassen in den Wahrnehmungsprozess geschieht ernsthaft und ohne Druck.

- Das gemeinsame Wahrnehmen entwickelt sich, ohne den/die AndereN zu überzeugen oder zu überreden.

- JedeR kann sagen, was er/sie wahrnimmt, denkt und fühlt und was der Prozess auslöst. Er/sie teilt sich mit, ohne jemanden verändern zu wollen.

- Es gilt, die vielen und fremden Wahrnehmungen stehen lassen zu können. Forschung und Praxis produzieren Wahrheiten und keine davon ist absolut.

- Den oben genannten Richtlinien wird grundsätzlich zugestimmt oder sie werden sinnvoll verändert.

Entscheidend für die Akzeptanz dieser Regeln ist, wie glaubwürdig ich als Gruppenleiterin sie vorlebe. Denn ein wesentlicher Konflikt zwischen den Menschen besteht laut Buber darin, dass ich nicht sage, was ich meine und dass ich nicht tue, was ich sage. Durch meine Wahrhaftigkeit kann ich Vertrauen in den Lernprozess wecken und fördern. Scheinheiligkeit ist Gift für den Dialog, lässt Beziehung verfallen und verstärkt den Glauben, nur potentielles Herrschaftsgebaren sei realistisch und die Wirklichkeit. Doch vergrößert eine solche Sichtweise die Welt des Misstrauens. Die dahinter liegende Krise zeigt sich im Verhältnis des Menschen zur Sprache und zum Gespräch: Der Mensch traut sich nicht mehr dem Gespräch an, weil die Voraussetzung, das Vertrauen verloren gegangen ist. Die Dialoggruppe ist deswegen als ein Gesprächskreis $\mathrm{zu}$ verstehen, in dem die Teilnehmer/innen die dialogischen Grundworte Ich-Es und Ich-du sprachlich ausdrücken können. Denn 
„Grundworte sagen nicht etwas aus, was außer ihnen bestünde, sondern gesprochen stiften sie einen Bestand. Grundworte werden mit dem Wesen gesprochen. Wenn Du gesprochen wird, ist das Ich des Wortpaars Ich-Du mitgesprochen. Wenn Es gesprochen wird, ist das Ich des Wortpaars Ich-Es mitgesprochen. Das Grundwort Ich-Du kann nur mit dem ganzen Wesen gesprochen werden. Das Grundwort Ich-Es kann nie mit dem ganzen Wesen gesprochen werden“ (Buber 1995, S. 3, erster Teil: 2.*Abschnitt ${ }^{11}$ ).

So lernen die Teilnehmer/innen ihre Wahrnehmungen sprachlich mitzuteilen und durch das Erzählen bekommen ihr Sein und ihre Wahrnehmungen in der Gruppe „Bestand“ und die „,begriffslose Praxis“ Worte (vgl. Göppner 2009).

\section{Intentionale Inhalte einer transkulturellen Dialoggruppe}

Auch wenn Buber immer wieder verneint hat, dass er keine Lehre hat, sondern ein Gespräch führt, versuche ich sein Dialogisches Prinzip zu vermitteln. Insbesondere sind in einer transkulturellen Dialoggruppe zum interkulturellen Lernen und Forschen vier Intentionen zum Verstehen wichtig. Sie unterstützen die Teilnehmer/innen darin, die dialogische Haltung von der monologischen Beobachtung zu unterscheiden.

Demzufolge liegt die erste Intention in der differenzierenden Wahrnehmung zwischen dem Ich der monologischen Haltung und dem Ich der dialogischen Haltung. Buber beschreibt diese Ichs einerseits mit „Eigenwesen“ und andererseits mit „Person“ (Buber 1995a, S. 60-63, zweiter Teil: 9.*Abschnitt, vgl. Muth 2011, S. 76ff.). So bleibt das Ich des Eigenwesens bei sich, und als „Subjekt" macht es sein Gegenüber zum Es, zum Objekt. Das Ich der Person wird „Subjektivität“, „steht in der Beziehung" und sagt Du:

„Wer Du spricht, hat kein Etwas zum Gegenstand. Denn wo Etwas ist, ist anderes Etwas, jedes Es grenzt an andere Es, Es ist nur dadurch, dass es an andere grenzt. Wo aber Du gesprochen wird, ist kein Etwas. Du grenzt nicht" (Buber 1995a, S. 4f., erster Teil: 5.*Abschnitt).

Hier wird deutlich, warum uns für berührende Begegnungen zuweilen die Worte fehlen. Gerade, weil der Dialog, wenn er passiert, grenzenlos ist, fehlen genaue Begriffe. Dennoch haben wir Menschen eine Resonanz dafür. Mit Bubers Beschreibungen können wir immerhin für die dialogische Wirklichkeit ein begriffliches Gewahrsein entwickeln.

11 Da unterschiedliche Ausgaben von Bubers Schrift „Ich und Du“ vorliegen und das Werk keine Nummerierung einzelner Abschnitte hat, habe ich zu deren genauen Finden die jeweiligen Sternchen-Abschnitte abgezählt und angegeben. 
Die zweite Intention verwirklicht sich im Wahrnehmen der von Buber so genannten ,sechs Geister“, die den Dialog verhindern (vgl. Buber 1962a, S. 274, vgl. Muth 2011, S. 60ff.). Demzufolge können sechs Bilder, die ein Ich$\mathrm{Du}$ verbergen, wahrgenommen werden, wenn ich als Eigenwesen mein Gegenüber beobachte und in eine Beziehung zu ihm treten wünsche: Jedes beteiligte Ich hat ein Bild von sich selbst, sozusagen wie das Ich sich in seiner Selbstwahrnehmung erscheint. Es ist das Selbstbild. Dabei mache ich mich selbst zum Gegenstand. Ich befinde mich dann im Ich-Es-Verhältnis zu mir selbst. Zudem macht sich jedes Ich ein Bild vom Gegenüber, ein Fremdbild vom Du. Das ergibt ein weiteres Ich-Es-Verhältnis. Ich mache das Gegenüber in Form eines Bildes zum Es, zu einem Gegenstand, den ich beobachten kann. Genauso handelt mein Gegenüber. Schließlich unterstellt jedes beteiligte Ich dem Gegenüber, das er oder sie sich ein Bild über das Gegenüber macht, d.h. ein projiziertes Fremdbild, das nicht mit dem eigenen Selbstbild übereinstimmen muss, denn es ist mein entworfenes Bild, das ich ihm unterschiebe, das er oder sie von mir gemacht hat.

Bei einem Treffen von zwei Menschen ergeben sich sechs Bilder bzw. Geister in Ich-Es-Formen, die aber immer noch keine transkulturelle Begegnung zwischen Subjekten sind. Das wirkliche Du liegt aber jenseits interkultureller (Vor-)Urteile. Ich kann nur im echten Dialog herausfinden, wie sich mein Gegenüber und ich von den sechs Bildern unterscheiden. Und selbst diese Unterscheidungen sind diskontinuierlich, da sowohl das Ich-Du als auch die Bilder sich verändern können. In einem solchen Differenzierungsprozess können Teilnehmer/innen Pluralität hautnah erfahren. Durch das Bewusstwerden der sechs Geister werden Wahrnehmungen, die damit verbundenen Weltbilder, Theoriekonstruktionen und der darin implizite Wahrheitsglaube relativiert.

Die dritte Intention realisiert sich in der unterscheidenden Wahrnehmung zwischen Innewerden und Beobachten (vgl. Buber 1962b, S. 181ff., vgl. Muth 2011). Letztere Handlung geschieht als Ich-Es-Verhältnis. Innewerden hingegen drückt eine Ich-Du-Beziehung aus und meint ein Gewahrwerden. Als Gegenstand betrachtet, ist Innewerden eine Form von Wahrnehmung. Es geht dabei um ein Gewahrsein für die Situation, an der Ich und Du beteiligt sind und sich gegenseitig verantworten. JedeR spürt sich und ist für das Gegenüber auch achtsam. Wiederum bemühe ich Bubers (1962, S. 182) Aussage dazu im Original:

„Anders geht es zu, wenn mir ... ein Mensch begegnet, an dem mir etwas, was ich gar nicht gegenständlich zu erfassen vermag, etwas sagt. ... mir etwas zuspricht, mir etwas in mein eigenes Leben hineinspricht. ... Der Mensch selber in seinem Verhalten zu mir hat mit diesem Sagen nichts zu schaffen ... Nicht er sagt es mir .... es sagt.“ 
Das Zitat unterstreicht, wie einzigartig solche Momente sein können. Sie sind existentiell, liegen dennoch jenseits von Raum und Zeit und machen die Subjektivität von Menschen erlebbar. Zum Innewerden gehört konzeptionell das Erkennen von „Rückbiegung“. Sie ist das Gegenteil vom Gewahrwerden des Gegenübers. Rückbiegung schließt das Gegenüber vollständig aus. D.h.: Ich beobachte nicht einmal mehr den/die AndereN. Alles dreht sich um das eigene Ich. Das Du wird nur als eigenes Erlebnis gesehen bzw. dadurch übersehen. Es verschwindet im Ich, ohne jemals wirklich wahrgenommen worden zu sein.

Die vierte Intention beschreibt die professionelle Haltung von Forscher/innen und Pädagog/innen, wenn sie transkulturelle Prozesse initiieren und derer innewerden. Buber nennt die Haltung „einseitige Umfassung“" (1962c, S. 800ff., vgl. Muth 2011, S. 70ff.). Mit diesem Begriff soll die Einstellung der Professionellen, die transkulturell ausgerichtet sind, von denen, die interkulturellen Analysen betreiben, unterschieden werden. Die interkulturellen Forscher/innen beobachten im Ich-Es-Verhältnis. Forschung im Ich-Du-Modus hingegen meint ein zusätzliches ,wahrhaftes Einander-Umfassen" der beteiligten Menschen und fordert immer wieder das Aufgeben der Beobachter/in-Position. Mit anderen Worten: Die forschende Person pendelt fortlaufend zwischen Distanz und Beziehung, zwischen IchEs und Ich-Du (vgl. Muth/Nauerth 2008, S. 22). Steht der/die Forscher/in der Beziehung, erwartet sie/er nicht, eine echte wechselseitige Beziehung, denn es geht bei der transkulturellen Forschung nicht allein um die Subjektivität der Forschenden, sondern um das dialogische Geschehen für das Du, für das das Gewahrsein entwickelt wird. Der Gewahrseinsprozess beginnt mit der forschenden Person (vgl. Muth 2012b). Im Mittelpunkt steht jedoch die Beziehung, also das, was zwischen Ich und Du geschieht. Die Forschenden wollen das Ich-Du wecken und verstehen sich als Partner/innen der gemeinsamen Situation. Die/der Forscher/in steht damit nicht nur

,an seinem Pol der bipolaren Beziehung, sondern auch mit der Kraft der Vergegenwärtigung am anderen Pol und (muss) die Wirkung seines eigenen Handelns erfahren“" (Buber 1995b, S. 127).

Ein solcher Gewahrseinsprozess bleibt allein die Aufgabe der forschenden Personen, was die Umfassung der Begegnung zu einer einseitigen macht. Die weiteren Dialogpartner/innen der zu erforschenden Situation brauchen diese besondere Sichtweise nicht zu entwickeln. Entsprechend bieten forschende Dialogpädagog/innen ihr Ich an und sprechen Du, doch sind sie ,entrückt“:

„Jedes Ich-Du-Verhältnis innerhalb einer Beziehung, die sich als ein zielhaftes Wirken des einen Teils auf den anderen spezifiziert, besteht kraft einer Mutualität, der es auferlegt ist, keine volle zu werden“ (Buber 1995b, S. 127). 


\section{Was ist zu tun?}

Die einseitige Umfassung wird noch einmal erkennbar im pädagogischen Evaluieren einer Dialoggruppe, im Forschungsbericht oder am Ende eines Artikels. Hier werden die transkulturellen Begegnungen Objekte von Ich-EsErkenntnissen:

„Das einzelne Du muss, nach Ablauf des Beziehungsvorgangs, zu einem Es werden. Das einzelne Es kann, durch Eintritt in den Beziehungsvorgang, zu einem Du werden" (Buber 1995a, S. 33, erster Teil: 30.*Abschnitt, Hervorh. i. O.).

Das pädagogische wie das forschende Geschehen bekommen dadurch „Maß und Grenze“:

„Jedes Du in der Welt ist seinem Wesen nach verhängt, Ding zu werden oder doch immer wieder in die Dinghaftigkeit einzugehen. In der gegenständlichen Sprache wäre zu sagen: jedes Ding in der Welt kann, entweder vor oder nach seiner Dingwerdung, einem Ich als sein Du erscheinen. Aber die gegenständliche Sprache erhascht nur einen Zipfel des wirklichen Lebens" (Buber 1995a, S. 18, erster Teil: 22.*Abschnitt).

Mit abschließenden Worten: Transkulturelle Dialogik hier in Worten zu beschreiben, ohne eine Dialoggruppe zu erleben oder/und die Gegenwart des Dialogs im Sein selbst aufzudecken, mag für manche Leser/innen mehr Fragen als Antworten schaffen. Für andere mag dialogische Wirklichkeit über das Lesen meines Textes bewusster werden. Die dialogische Wahrheit dazu liegt im gelebten Leben:

„Was man nicht nachmachen kann: Der Kozker Rabbi sprach: ,Alles in der Welt kann man nachmachen, nur die Wahrheit nicht. Denn eine nachgemachte Wahrheit ist keine Wahrheit mehr“" (Buber 1949, S. 794).

\section{Literaturverzeichnis}

Buber, M. (1949): Die Erzählungen der Chassidim, Zürich

Buber, Martin (1962): Werke: Erster Band: Schriften zur Philosophie, MünchenHeidelberg

Buber, M. (1962a): Elemente des Zwischenmenschlichen, in: Martin Buber (1962): Werke: Erster Band: Schriften zur Philosophie, München-Heidelberg, S. 267-289

Buber, Martin (1962b): Zwiesprache, in: Martin Buber (1962): Werke: Erster Band: Schriften zur Philosophie, München-Heidelberg, S. 171-214

Buber, M. (1962c): Über das Erzieherische, in: Martin Buber (1962): Werke: Erster Band: Schriften zur Philosophie, München-Heidelberg, S. 787-808

Buber, Martin (1963): Antwort, in: Paul Arthur Schilpp/Maurice S. Friedman (Hg.): Martin Buber, Stuttgart, S. 589-639 
Buber, Martin (1995a): Ich und Du, Stuttgart

Buber, Martin (1995b): Nachwort, in: Martin Buber (1995a): Ich und Du, Stuttgart, S. 117-130

Deleuze, Gilles/Guattari, Felix (1977): Rhizom, Berlin

Göhlich, Michael (2009): Anders Sein - Zur existentiellen Bedeutung von Transkulturalität, in: Leopold Klepacki/Andreas Schröer/Jörg Zirfas (Hg.): Der Alltag der Kultivierung. Studien zu Schule, Kunst und Bildung, Münster, S. 137-148

Göppner, Hans-Jürgen (2009): „Unbegriffene Theorie - begriffslose Praxis“ - Sozialarbeitswissenschaft zwischen Wissenschaftstheorie, Programmierung des praktischen Handelns und Adressatennutzen, in: Bernd Birgmeier/Erik Mührel (Hg.): Die Sozialarbeitswissenschaft und ihre Theorie(n): Positionen, Kontroversen, Perspektiven, Wiesbaden, S. 245-256

Mührel, Erik (2009): Die Begründung der Sozialarbeitswissenschaft in den Sozialwissenschaften. Eine theoretische Reflexion. in: Bernd Birgmeier/Erik Mührel (Hg.): Die Sozialarbeitswissenschaft und ihre Theorie(n): Positionen, Kontroversen, Perspektiven, Wiesbaden, S. 257-267

Muth, Cornelia (2005a): Integrale Aspekte des Dialogischen Prinzips bei Martin Buber. in: Cornelia Muth: Willst Du mit mir gehen, Licht und Schatten verstehen? Eine Studie zu Martin Bubers Ich und Du, Stuttgart, S. 85-103

Muth, Cornelia (2005b): Die praktische Umsetzung des Dialogischen Prinzips nach Martin Buber, in: Kontemplation und Mystik 2, S. 5-11

Muth, Cornelia (2008): Dialogpädagogische Reflexion über transkulturelle Erwachsenenbildung in Aktion, in: Gruppendynamik und Organisationsberatung 4, S. 443 453

Muth, Cornelia (2009a): How to teach intersubjectivity, in: Journal of Social Practice 2, S. 201-213

Muth, Cornelia (2009b): Dialogische Pädagogik: Identitätsbildung durch die Andersheit, in: Ursula Walkenhorst/Annette Nauerth/Inge Bergmann-Tyacke/Kordula Marzinzik (Hg.): Kompetenzentwicklung im Gesundheits- und Sozialbereich, Bielefeld, S. 35-45Muth, Cornelia/Nauerth, Annette (Hg.) (2007): Dialog und Diagnostik. Ein praxisorientiertes Handbuch für Lehrende, Wien

Muth, Cornelia (2010): Respekt vor der Andersheit statt Beschämung, in: Cornelia Muth/Annette Nauerth (Hg.): Vertrauen gegen Aggression. Das Dialogische Prinzip als Mittel der Gewaltprävention, Schwalbach/Ts., S. 124-138

Muth, Cornelia (2011): Erwachsenenbildung als transkulturelle Dialogik, Schwal$\mathrm{bach} / \mathrm{Ts}$.

Muth, Cornelia (2012a): Von der interkulturellen Erfahrung zur transkulturellen Begegnung, Stuttgart (im Erscheinen)

Muth, Cornelia (2012b): Phänomenologische Praxisentwicklungsforschung, Stuttgart

Thürmer-Rohr, Christina (2003): Dialog und Dialogisches Denken in: Renate Niekant/Uta Schuckmann (Hg.): Feministische ErkenntnisProzesse. Zwischen Wissenschaftstheorie und politischer Praxis, Opladen, S. 231-256 